Romero Romero, D., Romero Morato, A, Guerrero Álvarez, J.J., Giménez de Azcarate Fernández, F., Cáceres Clavero, F., Moreira Madueño, J.M. (2016): "Análisis comparativo entre la distribución de usos del suelo y su accesibilidad visual”, GeoFocus (Artículos), no 20, p. 63-85. ISSN: 1578-5157 http://dx.doi.org/10.21138/GF.516

\title{
ANÁLISIS COMPARATIVO ENTRE LA DISTRIBUCIÓN DE USOS DEL SUELO EN ANDALUCÍA Y SU ACCESIBILIDAD VISUAL. REDIAM
}

\author{
DANIEL ROMERO ROMERO ${ }^{1}$, ANDRÉS LUIS ROMERO MORATO ${ }^{1}$, JUAN JOSÉ \\ GUERRERO ÁLVAREZ ${ }^{2}$, FERNANDO GIMÉNEZ DE AZCARATE FERNÁNDEZ ${ }^{2}$, \\ FRANCISCO CÁCERES CLAVERO ${ }^{3}$, JOSÉ MANUEL MOREIRA MADUEÑO ${ }^{3}$. \\ ${ }^{1}$ RQUER Tecnología y Sistemas, S.L. C/Cristo del Buen Fin, 7, 41002 Sevilla, España \\ dromero@rquertys.es, aromero@rquertys.es \\ ${ }^{2}$ Agencia de Medio Ambiente y Agua. Consejería de Medio Ambiente y Ordenación del Territorio. \\ Junta de Andalucía. Calle Johann G. Gutenberg 1, 41092 Sevilla, España \\ jiguerrero@agenciamedioambienteyagua.es, fgimenezdeazcarate@agenciamedioambienteyagua.es \\ ${ }^{3}$ Servicio de Evaluación y Análisis Ambiental (Viceconsejería). Consejería de Medio Ambiente y \\ Ordenación del Territorio de la Junta de Andalucía. \\ Avda. Manuel Siurot 50, 41071 Sevilla, España \\ francisco.caceres@juntadeandalucia.es, josem.moreira@juntadeandalucia.es
}

\section{RESUMEN}

La Red de Información Ambiental de Andalucía (REDIAM) dispone de cartografía multitemporal y a distinto nivel de detalle sobre usos y coberturas del suelo, así como de otros aspectos físicos que conforman la piel visible del territorio. Del mismo modo cuenta con capas producidas a través del Sistema de Visibilidad de Andalucía (SVA), relativas a la accesibilidad visual, es decir, que estiman el impacto sobre la percepción de los observadores de cualquier cambio sobre el territorio.

El objetivo del trabajo que aquí se presenta es un acercamiento estadístico entre usos y coberturas del suelo y accesibilidad visual, con el fin de obtener una comparativa -multiescalar y a distintos niveles de definición- entre los contenidos sustantivos del territorio y la consideración visual que la situación de estos produce en el observador genérico.

Palabras clave: visibilidad, SIOSE, usos del suelo, visor web, análisis de datos espaciales. 
Romero Romero, D., Romero Morato, A, Guerrero Álvarez, J.J., Giménez de Azcarate Fernández, F., Cáceres Clavero, F., Moreira Madueño, J.M. (2016): "Análisis comparativo entre la distribución de usos del suelo y su accesibilidad visual”, GeoFocus (Artículos), no 20, p. 63-85. ISSN: 1578-5157 http://dx.doi.org/10.21138/GF.516

\section{COMPARATIVE ANALYSIS BETWEEN LANDCOVER- LANDUSE AND VISUAL ACCESSIBILITY IN ANDALUSIA. REDIAM}

\section{ABSTRACT}

The Environmental Information Network of Andalusia (REDIAM on its Spanish acronim) has multitemporal mapping and differentiated levels of detail on uses and land cover, as well as other physical aspects that form the visible skin of the territory. Similarly, this network includes layers produced through the Visibility System of Andalusia (SVA), related to the visual accessibility, meaning that they estimate the impact on the observer perception regarding any change on the territory

The purpose of the work presented here is a statistical approach between land cover and visual accessibility, in order to obtain a comparative analysis-multi-scalar, and at different levels of definition - between the substantial contents of the territory (percentage of each coverage or land use) and the effect that the situation of these contents produces on the generic observer.

Keywords: visibility, SIOSE, land use, web- app viewer, spatial data analysis.

\section{Introducción.}

La actual perspectiva de cooperación interinstitucional, integración de la información ambiental y unificación de las infraestructuras (mediante estándares, directivas, etc.) es promovida a nivel europeo por la Comisión (CE) a través de la Agencia Europea de Medio Ambiente, la Red Europea de Información y Observación del Medio Ambiente (EIONET) y sus Centros Temáticos Europeos (CTE), y coordinada a través de sus Centros Nacionales de Referencia (CNR) y los Puntos Focales Nacionales y Autonómicos (PFN), entre los que se encuentra la REDIAM.

La integración de la información relativa al Medio Ambiente acometida en Andalucía desde la REDIAM se sigue proyectando en el diseño productos y servicios que además vienen con un especial valor añadido, la generación de conocimiento. Es en este escenario en el que se enmarca el trabajo abordado en este artículo, y cuyos primeros resultados han sido presentados (Romero et al., 2016). A partir de diversas fuentes de datos, se vienen desarrollando multitud de aplicaciones que tienen como último fin la difusión y la comunicación de la información a través de elaboraciones que los hagan comprensibles para un público amplio.

Tras la actividad productiva de la REDIAM subyace un complejo conjunto de líneas de trabajo cuya convergencia y miscibilidad en casos como el que aquí traemos, se propone no como paradigma en la generación y tratamiento de la información geográfica, sino como punto de partida para el desarrollo de instrumentos que ayuden a entenderla y aplicarla; en definitiva a calibrar el peso y la importancia que la misma tiene: 
Romero Romero, D., Romero Morato, A, Guerrero Álvarez, J.J., Giménez de Azcarate Fernández, F., Cáceres Clavero, F., Moreira Madueño, J.M. (2016): "Análisis comparativo entre la distribución de usos del suelo y su accesibilidad visual”, GeoFocus (Artículos), no 20, p. 63-85. ISSN: 1578-5157 http://dx.doi.org/10.21138/GF.516

1. Línea de reconocimiento biofísico y cartografía ambiental. La información relacionada con la ocupación del suelo nos permite cualificar el territorio atendiendo por un lado a su cobertura y a las propiedades biofísicas (Land-Cover) y por otro a la estructura productiva (LandUse). A nivel europeo el proyecto Corine Land Cover (CLC) tiene como objetivo obtener una base de datos sobre ocupación de suelo a escala 1:100.000, útil para el análisis territorial y la gestión de políticas europeas. En este plano, el Sistema Información sobre la Ocupación de Suelos de España (SIOSE) y el Sistema de Información sobre el Patrimonio Natural de Andalucía (SIPNA) son los proyectos nacionales y autonómicos referencia (con escala 1:25.000 y 1:10.000 respectivamente). $\mathrm{Su}$ desarrollo ha permitido la generación de una base de referencia óptima (a escala autonómica, pero fácilmente exportable a la escala nacional) para reunir en un único sistema de información los datos precisos para el conocimiento integral de la cobertura y uso del suelo, tanto en Andalucía como en España, con el propósito de 'recoger la información una vez' y 'utilizarla por todos', de acuerdo a los principios de la directiva INSPIRE de la Unión Europea (Gil et al. 2010).

2. Línea de desarrollo del Subsistema de Información del Paisaje, con el Sistema Compartido de Información sobre el Paisaje de Andalucía (SCIPA) y el Sistema de Visibilidad de Andalucía (SVA) como algunas de las iniciativas acometidas desde la REDIAM para canalizar y evaluar el grado de consecución de la Estrategia de Paisaje de Andalucía (EPA).

Desde antes de la formulación del Convenio Europeo del Paisaje (CEP, Consejo de Europa, 2000) la consideración de aspectos relacionados con la percepción visual y su influencia en las tareas de Ordenación del Territorio viene siendo una cuestión de difícil implementación. El carácter transversal y democrático del concepto "paisaje" provoca su irrigación en todas las políticas territoriales, no solo en las ambientales. Ello se traduce en la dificultad para elaborar criterios que, por un lado sean objetivos y reproducibles (Mérida, 1996) sin menoscabar lo dispuesto en el CEP en su artículo 1, que incorpora la percepción (y con ello la subjetividad) de la población como elemento clave en la definición de paisaje.

El SVA ha tratado de desmarcarse de la valoración subjetiva que supone el "paisaje" y su delimitación conceptual, aportando claves que ayuden a los expertos y gerentes en la toma de decisiones. Ello se ha traducido en el desarrollo de parámetros, algoritmos y métodos que permiten llevar a cabo el análisis pormenorizado de determinadas relaciones visuales geométricas existentes y potenciales, atendiendo a una red tupida de puntos de sampleado $(100 \mathrm{~m} \times 100 \mathrm{~m})$ y a las características morfológicas del territorio (Cáceres et al., 2014). Una de sus explotaciones se ha basado en el estudio de la Accesibilidad Visual comparativa de cada punto del territorio, con la que podemos estimar el grado de afección de una intervención humana atendiendo a datos específicos como puede ser la altura de la misma o el modelo tentativo de distribución de observadores por el territorio, obtenida igualmente a partir de SIOSE (Romero et al., 2014).

La ligazón del concepto "paisaje" con el resto de cuestiones territoriales y humanas, y el tratamiento de su manifestación visual son cuestiones ampliamente estudiadas por muchos autores (Aramburu Maqua et al., 1994). En el último cuarto de siglo, sobre todo las que afectan a valoraciones cuantitativas están siendo solventadas por las Tecnologías de Información Geográfica (TIG). Éstas se retroalimentan con los avances experimentados en los Sistemas de Información Geográfica (SIG) y en el análisis de datos espaciales. Las investigaciones en los SIG han mejorado nuestra capacidad técnica para manejar datos de referencia espacial estimulando las reflexiones 
Romero Romero, D., Romero Morato, A, Guerrero Álvarez, J.J., Giménez de Azcarate Fernández, F., Cáceres Clavero, F., Moreira Madueño, J.M. (2016): "Análisis comparativo entre la distribución de usos del suelo y su accesibilidad visual”, GeoFocus (Artículos), no 20, p. 63-85. ISSN: 1578-5157 http://dx.doi.org/10.21138/GF.516

sobre la relación entre la "realidad geográfica" y la conceptualización y representación (abstracción) de esa realidad en formas digitales finitas (Goodchild y Haining, 2005).

Las técnicas basadas en álgebra de mapas y el modelado cartográfico han facilitado el análisis espacial de los datos ráster provenientes de las diferentes capas empleadas utilizando distintos tipos de funciones (Olaya, 2011). Partiendo de la definición de operadores sencillos combinados a través de expresiones algebraicas es posible componer modelos más complejos (Tomlin, 1990 y 1994 y Berry, 1993). Haciendo uso de operaciones locales (tesela por tesela) se han relacionado las magnitudes asociadas al cálculo de la accesibilidad visual y la extensión superficial de las diferentes coberturas de suelo referidas a una versión simplificada de SIOSE. En el ámbito de los estudios del paisaje estas operaciones son empleadas normalmente para obtener valores sencillos asociados a cada tesela (pendiente, orientación, pertenencia a una cuenca hidrológica) y otros más complejos asociados a la relación geométrica entre dichas teselas y distintas localizaciones de observación (Guerrero et al., 2010) a partir de un Modelo Digital de Elevaciones (DEM).

\subsection{Encuadre y delimitación.}

\subsubsection{El marco espacial.}

El marco espacial de desarrollo del proyecto se ciñe a un conjunto de ámbitos de gestión administrativa reconocidos y publicados para la comunidad autónoma andaluza por el Instituto de Estadística y Cartografía de Andalucía (IECA). Cada uno de las escalas estudiadas tiene cobertura total sobre el territorio andaluz, existiendo cuatro niveles de desagregación, comunidad autónoma, provincias, comarcas y municipios. Las unidades de cada uno de los tres primeros delimitan perfectamente a un conjunto de las de orden inferior, yendo desde el nivel comunidad autónoma al nivel término municipal.

Estos entornos administrativos que se presentan pues, en distintos escalones de gestión territorial han sido extraídos del compendio de Datos Espaciales de Referencia de Andalucía para escalas intermedias -DERA, un repertorio de bases cartográficas de diferente naturaleza geométrica (puntos, líneas, polígonos, imágenes ráster) referidas al territorio andaluz. Se presenta en una veintena de bloques temáticos (relieve, hidrografía, redes viarias, divisiones administrativas, etc.) que permiten el acceso centralizado a información de muy distinta procedencia con garantía de actualización, coherencia geométrica y continuidad territorial. La información se encuentra accesibles por capas en formato shapefile (.shp), en sistema de referencia geodésico ETRS89 y proyectadas en UTM huso 30.

En el Grupo 17 del citado repertorio, "Divisiones administrativas", podemos encontrar la capa "límites de términos municipales" (da02_term_munic), a partir de la cual se determinan las comarcas según la agrupación que, con criterio turístico se da en la "ORDEN de 14 de marzo de 2003, por la que se aprueba el mapa de comarcas de Andalucía a efectos de la planificación de la oferta turística y deportiva". Finalmente, se realiza la agrupación por provincias, acabando en la obtención por agregación del límite de la Comunidad Autónoma (figura 1). 
Romero Romero, D., Romero Morato, A, Guerrero Álvarez, J.J., Giménez de Azcarate Fernández, F., Cáceres Clavero, F., Moreira Madueño, J.M. (2016): "Análisis comparativo entre la distribución de usos del suelo y su accesibilidad visual”, GeoFocus (Artículos), no 20, p. 63-85. ISSN: 1578-5157 http://dx.doi.org/10.21138/GF.516

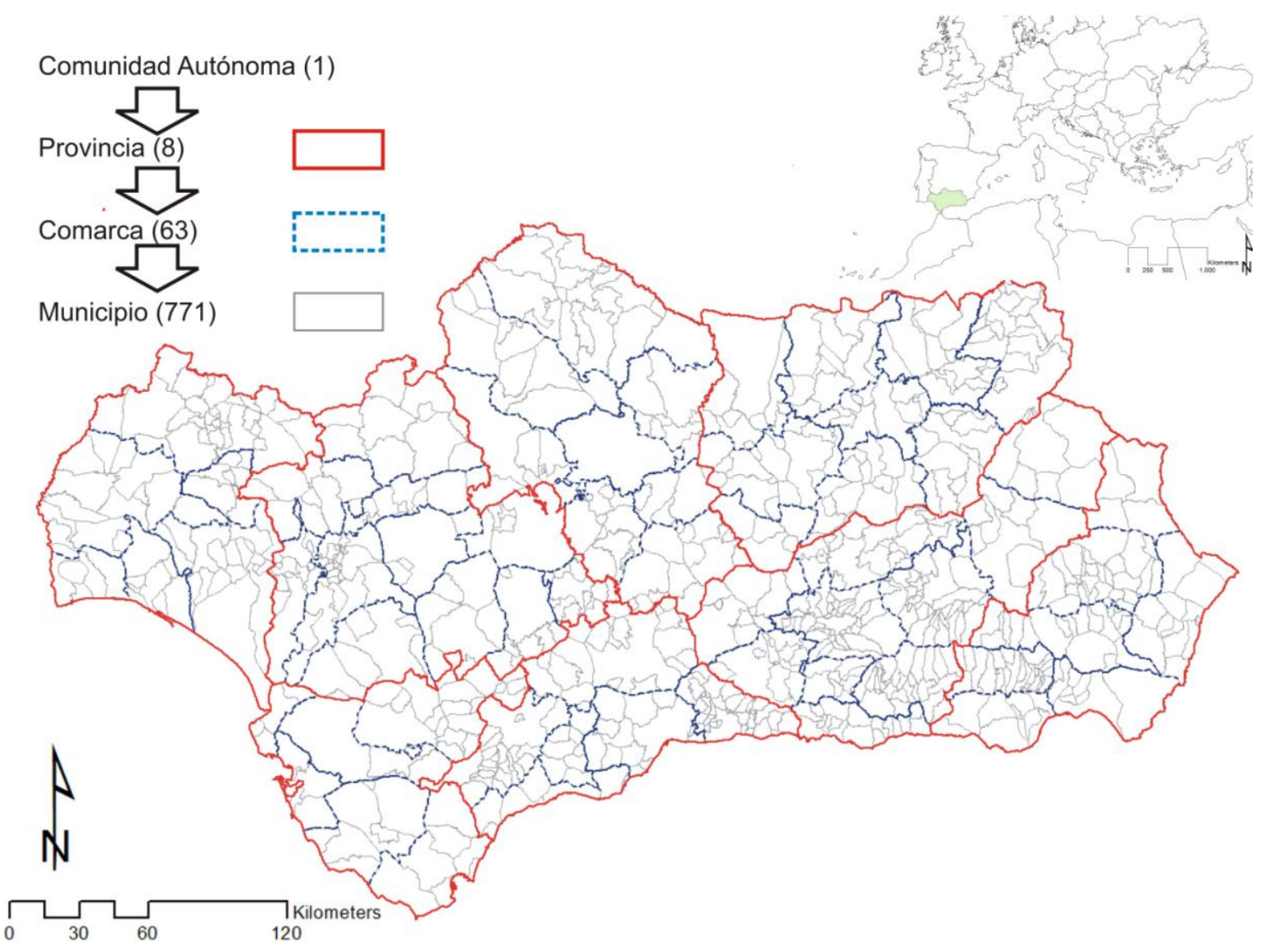

Figura 1. Unidades administrativas de gestión.

Fuente elaboración propia sobre datos del DERA.

\subsubsection{El marco temporal.}

El proyecto se encuadra temporalmente en una de las acciones acometidas desde la REDIAM y la Consejería de Medio Ambiente y Ordenación del Territorio de la Junta de Andalucía durante el año 2013, "Análisis de la realidad territorial vs paisaje percibido", con el fin de alcanzar uno de los objetivos de calidad paisajística trazados desde la Estrategia de Paisaje de Andalucía, el de fomentar y potenciar la sensibilización, la educación y la formación en materia de paisaje (Objetivo 7). La concreción de estos objetivos está directamente relacionada con el desarrollo de líneas de acción estratégica en las que se reconoce cada proyecto. La asociada al proyecto presentado (Educación, formación e investigación y proyectos de cooperación) contienen un valor añadido de especial relevancia en la política de paisaje, basado en hacer duradera la responsabilidad e implicación de la sociedad y que requiere, asimismo favorecer mecanismos de confluencia y coherencia entre las distintas instancias que han de intervenir en estos planteamientos que tienen un marcado carácter horizontal y transversal. 
Romero Romero, D., Romero Morato, A, Guerrero Álvarez, J.J., Giménez de Azcarate Fernández, F., Cáceres Clavero, F., Moreira Madueño, J.M. (2016): "Análisis comparativo entre la distribución de usos del suelo y su accesibilidad visual”, GeoFocus (Artículos), no 20, p. 63-85. ISSN: 1578-5157 http://dx.doi.org/10.21138/GF.516

\subsection{Objetivo metodológico.}

Se trata de obtener, para distintos niveles autocontenidos y jerarquizados, relacionados con la gestión y que cubren la totalidad de la Comunidad Autónoma de Andalucía, estadísticas por un lado sobre la ocupación y uso del suelo basadas en clases generadas a partir de modelos de datos como los del Sistema de Información sobre el Patrimonio Natural de Andalucía) SIPNA y SIOSE, también jerarquizadas y autocontenidas. Esto permitirá que a partir del nivel de desagregación máximo se pueda llegar a consolidar de forma sencilla estadísticas básicas en niveles de agregación mayores (tanto en el sentido geográfico del término - de comunidad autónoma a término municipalcomo en el temático - de matorral a forestal). Por otra parte, es posible referir al mismo marco espacial, constituido por los niveles anteriormente expuestos, las estadísticas relativas a la accesibilidad visual del territorio. De esta manera será posible siempre comparar la importancia territorial (en términos de porcentaje sobre la superficie de la unidad de gestión que sea) de un uso o cobertura con su importancia perceptual (es decir, qué importancia adquiere en la visión del ciudadano).

\section{Metodología}

\subsection{Estado de la cuestión}

Con los datos ofrecidos por el SVA sobre visibilidad efectiva y potencial, y a través de su combinación con otros derivados de la interpretación realizada sobre distintas bases de referencia para realizar una segmentación del territorio sobre la que inferir una distribución de observadores tentativa y consensuada, ha sido ya posible obtener una aproximación objetiva y replicable a la Accesibilidad Visual del territorio, entendida como "la afección que produciría una intervención genérica, en la visión de la totalidad de los observadores potenciales, de forma que conociendo su localización y altura previstas se obtenga un valor sintético mediante el cual comparar diferentes alternativas".

Se realizó una versión teniendo en cuenta los efectos que distancia y proyección visual (Domingo- Santos 2011) tienen en la alteración de la imagen, a la que se llamó psicofísica ('psi'), y otra en la que no se tienen en cuenta estos efectos ('sim'), útil para poder establecer comparativas y para intervenciones territoriales, cuyo efecto visual se pueda entender que no pierde importancia con la distancia. Esta comparativa nos sirvió para demostrar con facilidad el efecto amortiguador de la distancia sobre el potencial impacto que éstas producen.

Una primera solución en esta aplicación, que presuponía una distribución homogénea de observadores y a la que se le denominó Intervisibilidad (IVS), ofrecía para cada tesela (según la resolución del modelo ráster, en el caso del SVA de 20 metros) del territorio andaluz, la suma de observadores que pueden verla. A través de los resultados de IVS se pudieron elaborar estadísticas e indicadores como la media (en porcentaje) de visibilidad real sobre el total posible para cada punto o conjunto de puntos de observación considerados, basándose en conceptos ya estudiados como el estudio de la cuenca visual relativa (Aguiló, 1981). La superficie máxima visible para cada localización era 70.686 has, área de un circulo de $15 \mathrm{~km}$ de radio, que es distancia máxima de visibilidad establecida en el SVA. Los puntos de sampleado estaban distribuidos regularmente por 
Romero Romero, D., Romero Morato, A, Guerrero Álvarez, J.J., Giménez de Azcarate Fernández, F., Cáceres Clavero, F., Moreira Madueño, J.M. (2016): "Análisis comparativo entre la distribución de usos del suelo y su accesibilidad visual”, GeoFocus (Artículos), no 20, p. 63-85. ISSN: 1578-5157 http://dx.doi.org/10.21138/GF.516

toda la superficie de la comunidad autónoma según una malla de 100x100m. A este indicador se le denominó Índice de Visibilidad Relativo (IVR).

El valor de esta información es mucho mayor en la medida en la que el SVA nos proporciona datos sobre la visibilidad potencial. Si bien lo anteriormente expuesto es entendible como la superficie vista desde cada punto de observación considerado, los valores de IVR para las alturas de intervención (Tabla 1 ) consideradas en el desarrollo del SVA ( 0 como la ya explicada, 10 , 20, 30,60 y 120 metros), pueden interpretarse como la superficie afectada o desde la que es visible un objeto de las alturas contempladas colocado en una posición genérica. Su media se reflejó por provincias y para la comunidad autónoma de Andalucía. Este acercamiento se entiende apropiado para estudios en los que el factor de distribución humana no parece importante, como el realizado sobre la influencia del relieve en la accesibilidad visual del territorio (Romero et al. 2014), o estudios en el ámbito de la arqueología (Wheatley 1995, Llobera, 2003 y Llobera et al. 2010).

Tabla 1. Índice de Visibilidad Relativo (IVR) para las provincias andaluzas sobre actuaciones genéricas de diferentes alturas

\begin{tabular}{|l|r|r|r|r|r|r|r|}
\hline \multirow{2}{*}{ Límite Administrativo } & \multirow{2}{*}{ Superficie (Has) } & \multicolumn{6}{|c|}{ IVR \% } \\
\cline { 3 - 8 } & & $\mathbf{0 ~ m . ~}$ & $\mathbf{1 0} \mathbf{~ m .}$ & $\mathbf{2 0} \mathbf{~ m . ~}$ & $\mathbf{3 0} \mathbf{~ m .}$ & $\mathbf{6 0} \mathbf{~ m .}$ & $\mathbf{1 2 0} \mathbf{~ m . ~}$ \\
\hline Almería & 876915 & 4,30 & 8,06 & 10,02 & 11,64 & 15,56 & 21,52 \\
\hline Cádiz & 744535 & 3,40 & 9,15 & 12,06 & 14,46 & 20,63 & 30,06 \\
\hline Córdoba & 1376899 & 1,97 & 4,86 & 6,97 & 8,94 & 14,41 & 23,63 \\
\hline Granada & 1263799 & 4,46 & 8,05 & 9,95 & 11,52 & 15,41 & 21,57 \\
\hline Huelva & 1015074 & 1,83 & 9,24 & 13,20 & 16,32 & 23,45 & 33,29 \\
\hline Jaén & 1348630 & 2,41 & 4,44 & 5,83 & 7,08 & 10,46 & 16,39 \\
\hline Málaga & 730749 & 3,63 & 6,63 & 8,35 & 9,79 & 13,35 & 18,95 \\
\hline Sevilla & 1404455 & 2,63 & 15,29 & 20,81 & 24,73 & 33,53 & 44,97 \\
\hline Andalucía & $\mathbf{8 7 6 1 0 5 6}$ & $\mathbf{3 , 0 8}$ & $\mathbf{8 , 2 2}$ & $\mathbf{1 0 , 9 0}$ & $\mathbf{1 3 , 0 6}$ & $\mathbf{1 8 , 3 5}$ & $\mathbf{2 6 , 3 0}$ \\
\hline
\end{tabular}

En una segunda hipótesis sin embargo, y en cada categoría de suelo (tabla 2, categorías de accesibilidad local) en la que habíamos seccionado el territorio (Guerrero et al. 2010) calculamos para cada punto, cuál sería la afección que produciría una intervención de cierta altura al conjunto de observadores potenciales situados en esta categoría. 
Romero Romero, D., Romero Morato, A, Guerrero Álvarez, J.J., Giménez de Azcarate Fernández, F., Cáceres Clavero, F., Moreira Madueño, J.M. (2016): "Análisis comparativo entre la distribución de usos del suelo y su accesibilidad visual”, GeoFocus (Artículos), no 20, p. 63-85. ISSN: 1578-5157 http://dx.doi.org/10.21138/GF.516

Tabla 2. Categorías de Accesibilidad Local y valores tomados para cada una de las explotaciones del SVA

\begin{tabular}{|l|l|r|r|}
\hline Categoría de Suelo & ABR & \multicolumn{2}{|c|}{ EXPLOTACIÓN } \\
\cline { 4 - 4 } & & Intervisibilidad & $\begin{array}{c}\text { Accesibilidad } \\
\text { Visual }\end{array}$ \\
\hline Núcleos urbanos de especial interés & & 1 & 500 \\
\hline Zonas residenciales & ZU & 1 & 200 \\
\hline Zonas urbanas no residenciales & ZR & 1 & 50 \\
\hline Suelos de dominante agrícola o alterado no urbano & ZN & RA & 1 \\
\hline Suelo de dominante forestal y natural & RF & 1 & 2 \\
\hline Mares y océanos & RO & 1 & 3 \\
\hline FF.CC & VF & 1 & 3000 \\
\hline Vías de gran capacidad & VA & 1 & 5000 \\
\hline Carreteras convencionales & VC & 1 & 2000 \\
\hline Itinerarios turísticos no rodados & VV & 1 & 10000 \\
\hline Alta frecuentación & TH & 1 & 5000 \\
\hline Baja frecuentación & TL & 1 & 1000 \\
\hline
\end{tabular}

Hay que subrayar que lo que se determina con estas operaciones es la cualidad que tiene cada localización de, situada sobre ella una intervención invariante, afectar la imagen del territorio que tienen los observadores situados en un determinado ámbito, caracterizado por contener una densidad de observadores diferente a otro. Los valores para cada provincia resultaban de sumar los asociados a las teselas recogidas en los límites de cada una de ellas, según cada una de las categorías de suelo (accesibilidad local) y para cada una de las alturas de intervención calculadas. En una última hipótesis derivada de esta, la determinación de la Accesibilidad Visual Ponderada (AVP), se consideraban conjuntamente todas las categorías de accesibilidad local, poniendo de relieve la probabilidad de encontrar observadores en un lugar respecto a otro. (Romero et al. 2014).

Con todo ello y tras el análisis de la accesibilidad visual, queda a la luz la necesidad de cruzar estos datos que se refieren a la percepción visual de nuestro entorno físico con otros que reflejen la realidad territorial. En este sentido, la piel del territorio puede quedar representada con la ayuda de algunos de los sistemas sobre ocupación y usos de suelo (SIOSE o SIPNA), mediante los cuales podemos conocer un valor referido a la superficie ocupada por cada una de las clases establecidas; además y yendo más allá se podría relacionar porcentualmente la realidad existente y lo que podíamos entender como percepción visual (AVP, $\mathrm{h}=0$ ); es decir proporcionar valores de AVP para cada una de las clases de suelo en los que el territorio queda seccionado. La Accesibilidad Visual Ponderada nos proporciona un valor para cada tesela igual al número de veces que dicha tesela es vista ( si colocásemos un objeto de altura definida, en este caso $\mathrm{h}=0$ ), desde el conjunto de puntos de observación considerados (en el SVA, una malla de observadores regular de $100 \mathrm{~m} \times 100 \mathrm{~m}$ en toda la extensión de la comunidad autónoma), teniendo en cuenta de forma combinada y sobre aquellos, cuestiones inherentes a su posición, como la distancia y la proyección visual (Higashiyama y Shimono 1984); pero sobre todo se introduce el factor de la accesibilidad 
Romero Romero, D., Romero Morato, A, Guerrero Álvarez, J.J., Giménez de Azcarate Fernández, F., Cáceres Clavero, F., Moreira Madueño, J.M. (2016): "Análisis comparativo entre la distribución de usos del suelo y su accesibilidad visual”, GeoFocus (Artículos), no 20, p. 63-85. ISSN: 1578-5157 http://dx.doi.org/10.21138/GF.516

local que pondera los valores de las teselas percibidas en función de la probabilidad de encontrar observadores en una localización de observación respecto a otra.

A partir de aquí parece oportuno buscar un mismo espacio de relación que nos permita asociar los datos de visibilidad a los de ocupación de suelo, ya que si bien aquellos están referidos a teselas de $20 \mathrm{~m}$ x20m,-según el modelo ráster bajo el que se implementa el SVA, en el caso de SIOSE, los datos están referidos a entidades vectoriales (polígonos).

\subsection{Origen de los datos.}

Planteado este escenario, podemos apuntar hacia la preparación de un espacio de trabajo común en el que podamos cotejar y comparar:

1. Por un lado los datos sobre visibilidad referidos anteriormente. Se trata de datos relativos al modelo raster sobre el que se ha desarrollado la explotación de Accesibilidad Visual del SVA. Esto significa que los datos calculados están referidos a cada una de las teselas que componen dicho modelo, que tendrán un tamaño definido por su resolución (en el caso del SVA 20 metros). De todos los indicadores sobre los que se han calculado valores para toda la cobertura del territorio andaluz hemos seleccionado uno de ellos, elaborado con la intención de aproximar la percepción del territorio de una forma global, no teniendo en cuenta la posición de un observador, sino la de todos a la vez y una distribución tentativa de los mismos, en función de una clasificación del suelo relacionada con la probabilidad de encontrar observadores. Así la magnitud de referencia considerada es la Accesibilidad Visual Ponderada (AVP), tomando $\mathrm{h}=0$ como altura de intervención y teniendo en cuenta la afección psicofísica, valorando de forma conjunta la distancia y la proyección visual (Higashiyama y Shimono 1984).

El SVA nos proporciona a través de los mapas de Accesibilidad Visual Ponderada (para cada altura de intervención calculada) un valor escalar para cada tesela $(20 \times 20 \mathrm{~m})$ del territorio andaluz, indicador de cómo dicho territorio es percibido por la globalidad de observadores teniendo en cuenta cuestiones como la distancia de observación y la proyección visual, la altura de observación, la morfología del territorio, la facilidad de acceso a cada una de las localizaciones de observación, la actitud del observador que se encuentra en esa situación, la aptitud del terreno desde el que se observa, o la probabilidad relativa de encontrar observadores en un lugar respecto a otro (figura 2).
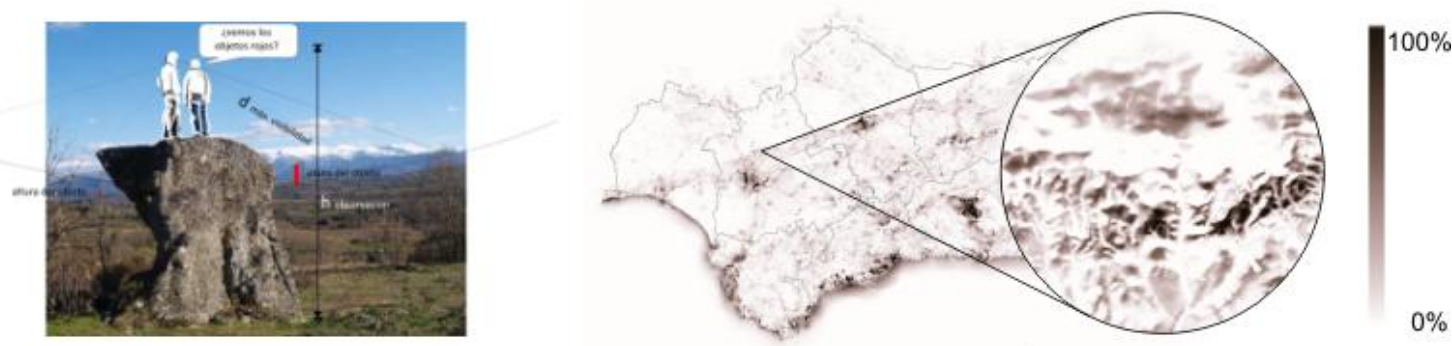

Figura 2. Accesibilidad Visual Ponderada para intervenciones de $0 \mathrm{~m}$ de altura, que puede interpretarse como cuál es la influencia de cada tesela de territorio en la imagen percibida por la población.

Fuente elaboración propia sobre datos de la REDIAM. 
Romero Romero, D., Romero Morato, A, Guerrero Álvarez, J.J., Giménez de Azcarate Fernández, F., Cáceres Clavero, F., Moreira Madueño, J.M. (2016): "Análisis comparativo entre la distribución de usos del suelo y su accesibilidad visual”, GeoFocus (Artículos), no 20, p. 63-85. ISSN: 1578-5157 http://dx.doi.org/10.21138/GF.516

2. Por otra parte los datos sobre la cobertura del suelo, los que conforman su piel externa, se encuentran vinculados a modelos de datos vectoriales, es decir que los datos están referidos a entidades espaciales vectoriales (puntos, líneas, polígonos). Su entidad de trabajo es el polígono, que es la unidad espacial que presenta una ocupación de suelo con cobertura homogénea. Será fundamental, de cara a reflejar cómo se distribuye el suelo de nuestra comunidad autónoma a efectos de uso y coberturas, concretándose en un mapa continuo, y con gran nivel de detalle.

\subsection{Metodología de análisis.}

\section{A) Paso 1: Rasterización de la capa SIOSE-OS.}

Si un ráster es, en esencia, cualquier tipo de imagen digital representada en mallas, su modelo asociado se centra en las propiedades del espacio más que en la precisión de la localización. Divide el espacio en celdas regulares donde cada una de ellas representa un único valor. Así, se trata de un modelo de datos muy adecuado para la representación de variables continuas en el espacio. Entendemos a partir de este momento que la solución al problema planteado podría pasar por usar como base un modelo ráster de la misma resolución que el usado para las explotaciones de accesibilidad visual del SVA, 20 metros de resolución, que quiere decir que el tamaño del píxel que adopta los distintos valores determinados es de $20 \mathrm{~m} \times 20 \mathrm{~m}$.

El objetivo de esta fase fue obtener, para la extensión de la Comunidad Autónoma, los valores porcentuales de cada una de las coberturas SIOSE simplificadas. Estas son las que figuran en la tabla 3:

Tabla 3. Clasificación de las coberturas SIOSE OS simplificadas

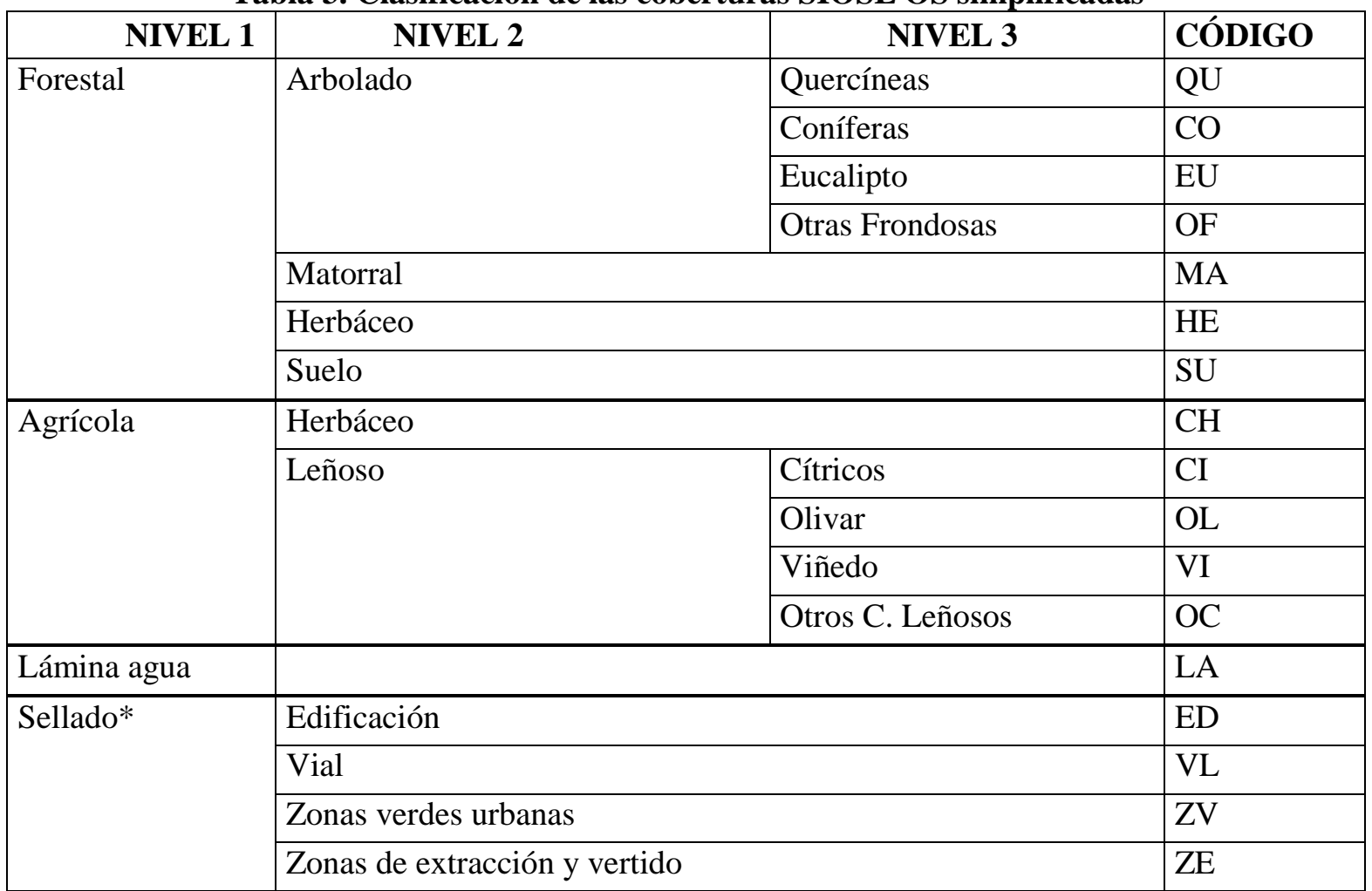

*Sellado incluye suelos muy alterados (verdes urbanas y zonas de extracción y vertido). 
Romero Romero, D., Romero Morato, A, Guerrero Álvarez, J.J., Giménez de Azcarate Fernández, F., Cáceres Clavero, F., Moreira Madueño, J.M. (2016): "Análisis comparativo entre la distribución de usos del suelo y su accesibilidad visual”, GeoFocus (Artículos), no 20, p. 63-85. ISSN: 1578-5157 http://dx.doi.org/10.21138/GF.516

El resultado esperado es entonces 17 rasters con valor porcentual, uno por cada una de las clases de suelo de nivel finalista (aquellas que tienen código).

La opción metodológica más directa se basó en un principio, en rasterizar directamente la capa vectorial SIOSE OS (SIOSE 2009 simplificado), tomando como valor de referencia el que refleja el campo correspondiente. No obstante este método provoca problemas a la hora de obtener valores de suma para localizaciones concretas, puesto que cada rasterización se hacía por separado, provocando desajustes (por exceso o defecto) en localizaciones de límite de entidad vectorial.

Este efecto, que resulta poco importante cuando el tamaño de píxel es mucho más pequeño que los polígonos a rasterizar, es determinante en el caso de SIOSE, una base de referencia muy detallada geométricamente, con polígonos diferenciados a partir de $50 \mathrm{~m} 2$. Para entender más fácilmente este efecto, imaginemos que el tamaño de píxel con el que rasterizamos fuera muy grande, y los polígonos fueran muy pequeños.

El caso lo podemos ilustrar como aparece en la figura 3. Si transformamos por ejemplo el valor de 'Cultivos Herbáceos' $(\mathrm{CH})$ y hay un polígono que supone el $40 \%$ del área con el $0 \%$ de este valor, otro que supone el $30 \%$ del píxel con $100 \% \mathrm{CH}$ y un último que cubre el $30 \%$ restante del píxel con un $95 \%$ de $\mathrm{CH}$, el valor que tomará el píxel para las últimas dos opciones será $0 \%$, cuando el valor real del píxel sobrepasa el 50 \% de Cultivos Herbáceos.

Para evitar este efecto, que en una cartografía de alto nivel de detalle como SIOSE y para un tamaño de píxel que no suponga archivos demasiado grandes, se opta por una metodología que pasa, en primer lugar, por hacer un análisis estadístico zonal para el área que cubrirá cada píxel del ráster (figura 3), para después determinar cuál es el valor que tomará el píxel en cada uno de esos rasters:
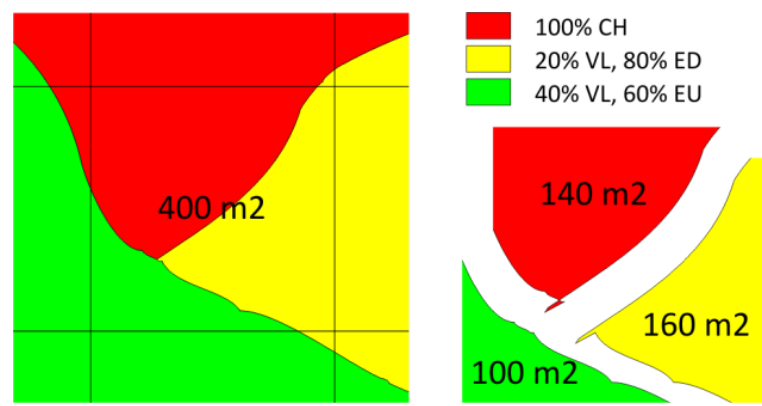

\begin{tabular}{|l|}
\hline $140 * 1=140 \mathrm{~m} 2 \mathrm{CH}$ \\
\hline $160 * 0.2=32 \mathrm{~m} 2 \mathrm{VL}$ \\
\hline $160 * 0.8=128 \mathrm{~m} 2 \mathrm{ED}$ \\
\hline $100 * 0.4=40 \mathrm{~m} 2 \mathrm{VL}$ \\
\hline $100 * 0.6=60 \mathrm{~m} 2 \mathrm{EU}$ \\
\hline $140 / 400=35 \% \mathrm{CH}$ \\
\hline$(32+40) / 400=18 \% \mathrm{VL}$ \\
\hline $128 / 400=32 \% \mathrm{ED}$ \\
\hline $60 / 400=15 \% \mathrm{EU}$ \\
\hline
\end{tabular}

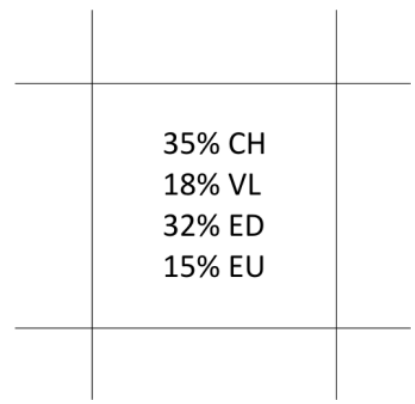

Figura 3. Análisis estadístico zonal para determinar los valores de píxel.

Fuente de elaboración propia

De esta forma, el píxel, que en el caso de optar por la primera metodología, tendría un contenido de $100 \% \mathrm{CH}$ (uso de punto central) o de $20 \% \mathrm{VL}$ (Vial), $80 \% \mathrm{ED}$, Edificación (uso de máxima área contenida -combinada o no), pasa a tener un contenido más complejo y que representa mucho mejor la realidad del territorio.

Obtenemos 17 rasters (figura 4) con cobertura para toda Andalucía, de tamaño de píxel 20x20 m. asociados a cada una de las coberturas reflejadas en la tabla 3. Los archivos comprimidos 
Romero Romero, D., Romero Morato, A, Guerrero Álvarez, J.J., Giménez de Azcarate Fernández, F., Cáceres Clavero, F., Moreira Madueño, J.M. (2016): "Análisis comparativo entre la distribución de usos del suelo y su accesibilidad visual”, GeoFocus (Artículos), no 20, p. 63-85. ISSN: 1578-5157 http://dx.doi.org/10.21138/GF.516

ocupan un tamaño en memoria [con tipo de datos 'float' (4b)] de 1.601.250.000 bytes, 1.6 Gb. Este tamaño, para esta resolución de píxel de $20 \mathrm{~m}$ resulta ser óptimo para poder manejar el ráster completo.

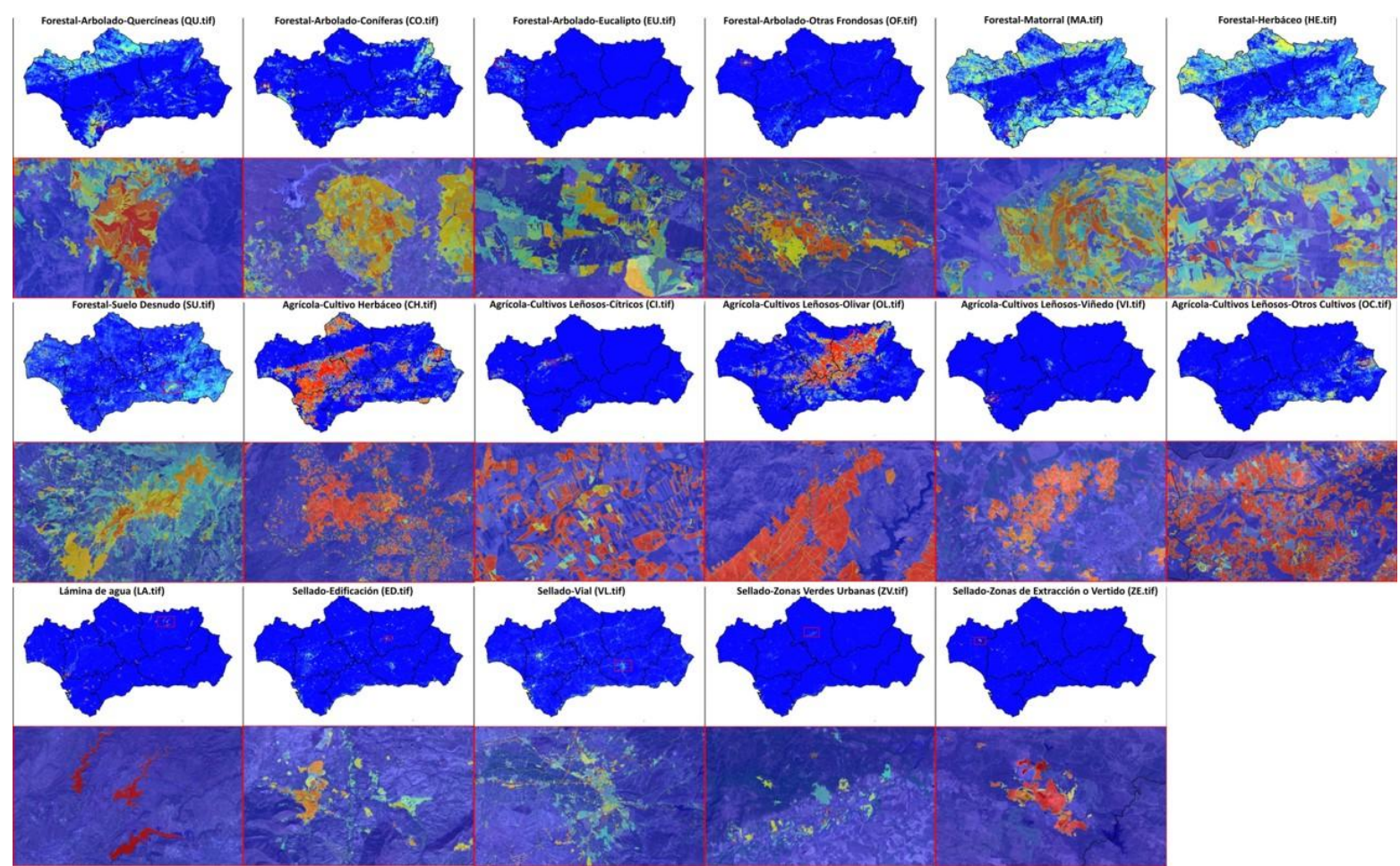

Figura 4. 17 rasters de coberturas SIOSE simplificadas.

Fuente de elaboración propia sobre datos de la REDIAM

B) Paso 2: Comparación entre la capa SIOSE-OS rasterizada y la capa Accesibilidad Visual Ponderada (AVP, con afección psicofísica- distancia y proyección visual, y $h=0)$.

Del mismo modo que hemos explicado con anterioridad, y una vez rasterizadas las coberturas SIOSE simplificadas y ajustadas a un mapa ráster de $20 \mathrm{~m}$ de resolución, podemos deducir el valor del área en $\mathrm{m} 2$ de cada una de las coberturas definidas para cada píxel y dentro de cada uno de los ámbitos de gestión. Es decir podemos saber qué superficie de coníferas hay en determinado término municipal, comarca o provincia, o qué cantidad de suelo destinado a cultivos de olivar hay en un determinado municipio, comarca o provincia, así con las 17 coberturas simplificadas.

Partimos pues de una versión del SIOSE con fecha de referencia 2009 que se simplifica a 17 clases básicas (SIOSE ocupación del suelo OS), y desde el que obtenemos, para cada píxel de la extensión de Andalucía, 17 valores porcentuales relativos a estas clases (figura 4). En definitiva, una cartografía con alto nivel de detalle geométrico para un tamaño de píxel de 20x20m y que permite la comparabilidad con los datos relativos a accesibilidad visual (Figura 5). 
Romero Romero, D., Romero Morato, A, Guerrero Álvarez, J.J., Giménez de Azcarate Fernández, F., Cáceres Clavero, F., Moreira Madueño, J.M. (2016): "Análisis comparativo entre la distribución de usos del suelo y su accesibilidad visual”, GeoFocus (Artículos), no 20, p. 63-85. ISSN: 1578-5157 http://dx.doi.org/10.21138/GF.516

\section{SIPNA '09 (\% sobre $\sim 10.000$ coberturas, usos y atributos) simplificación L \\ SIPNA '09 OS (\% sobre 17 coberturas fundamentales) rasterización}

17 ráster $20 \times 20 \mathrm{~m}$ representando $\%$ coberturas fundamentales

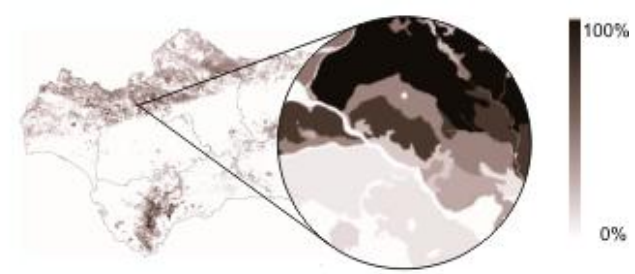

Figura 5. Proceso de simplificación y rasterización de la base de referencia SIOSE '09. Fuente de elaboración propia sobre datos de la REDIAM

Pero ¿Cómo asociamos los resultados obtenidos de Accesibilidad Visual Ponderada (AVP, $\mathrm{h}=0$, psi) y las 17 coberturas SIOSE simplificadas obtenidas? Usando herramientas sencillas es posible comparar cada una de las celdas de ambos ráster obtenidos. El geoprocesamiento admite la automatización de los cálculos sobre dichos valores al proporcionar un amplio 'set' de herramientas, y los mecanismos para combinarlas en un conjunto de operaciones, mediante modelos y secuencias de comandos. Su uso ha facilitado la elaboración de estadísticas sobre el valor de cada celda; esto es el valor porcentual que se obtiene de cada raster de cobertura SIOSE-OS simplificada y la Accesibilidad Visual Ponderada para dicha tesela (figura 6).

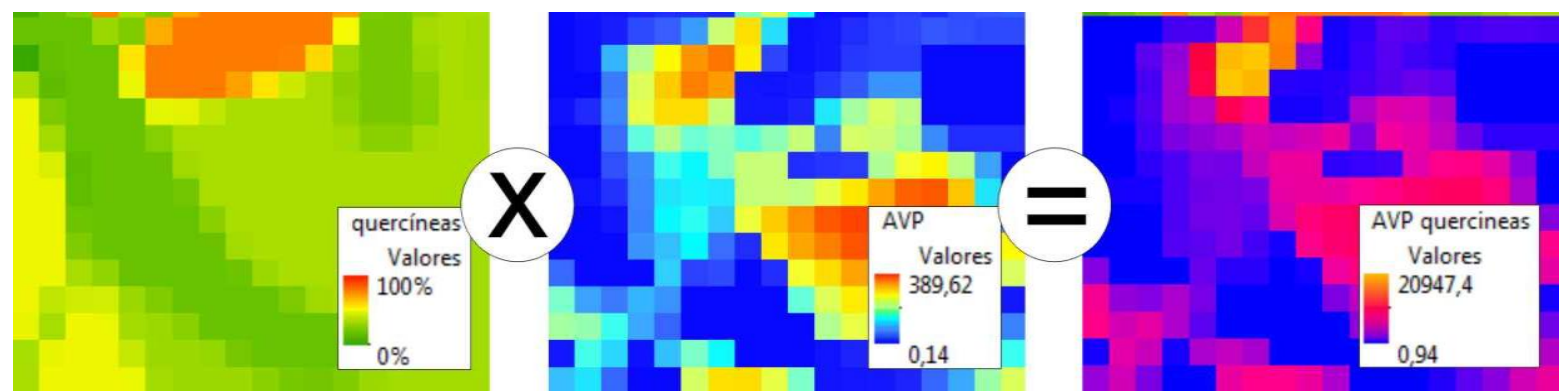

Figura 6. Ejemplo polígono rasterizado y superpuesto con Accesibilidad Visual Ponderada (AVP)

Fuente de elaboración propia.

Para cualquier ámbito en los que se ha seccionado el territorio andaluz, es posible asociar para cada píxel, los distintos valores de cada una de las 17 coberturas SIOSE simplificadas rasterizadas y su valor correspondiente de AVP.

La metodología desarrollada apunta hacia un entorno que permite comparar la superficie de cada una de las coberturas definidas y su accesibilidad visual. Esto se traduce en valores de superficie y valores de Accesibilidad Visual para cada una de las 17 clases SIOSE según las delimitaciones espaciales estudiadas. La suma de los parciales referidos a las distintas coberturas nos permite establecer valores totales sobre los que deducir porcentajes de ocupación y de visibilidad de cada cobertura.

Dichos valores son representados en dos coronas circulares concéntricas:

-Una exterior que nos presenta los cálculos de superficie de cada cobertura en el ámbito seleccionado y su porcentaje respecto a la superficie total de dicho ámbito. 
Romero Romero, D., Romero Morato, A, Guerrero Álvarez, J.J., Giménez de Azcarate Fernández, F., Cáceres Clavero, F., Moreira Madueño, J.M. (2016): "Análisis comparativo entre la distribución de usos del suelo y su accesibilidad visual”, GeoFocus (Artículos), no 20, p. 63-85. ISSN: 1578-5157 http://dx.doi.org/10.21138/GF.516

- Una interior que representa los cálculos de Accesibilidad Visual Ponderada (AVP, h=0, psi) de cada una de las coberturas y su porcentaje respecto al valor total de Accesibilidad Visual Ponderada del ámbito seleccionado

Las gráficas representadas (figura 7) nos mostrarán para todos los ámbitos de gestión y de una sola mirada los 'desequilibrios' o 'equilibrios' más significativos entre la superficie que ocupan las distintas coberturas de suelo SIOSE (la piel del territorio) y como son percibidas visualmente. Es posible en este sentido realizar análisis en las 4 escalas definidas.
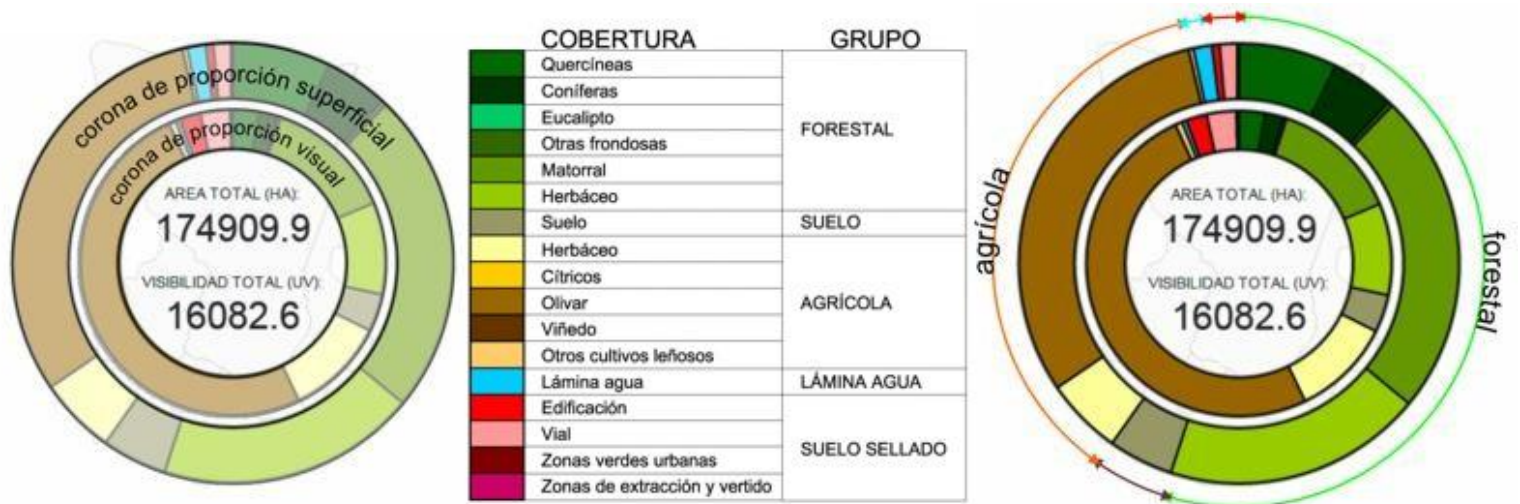

Figura 7. Descripción de las coronas gráficas de visualización de resultados

Fuente de elaboración propia.

\section{Descripción y análisis de resultados.}

3.1. Descripción de los resultados mediante las tablas comparativas.

Las estadísticas resultantes obtenidas a través de operaciones de geoprocesamiento compatibilizan la distinta naturaleza de las capas que se pretenden combinar de la forma más precisa posible, garantizando la comparabilidad de las series estadísticas que se obtienen para la descripción del territorio en cuanto a su proporción superficial con las obtenidas teniendo en cuenta la distribución heterogénea de la accesibilidad visual del territorio. El estudio de las tablas estadísticas por sí mismo puede ser muy revelador, pero resulta un documento un tanto complicado desde el punto de vista de la difusión a no expertos.

Los resultados han sido agrupados por unidades visuales y superficiales, pudiendo con ello inferir porcentajes sobre los valores absolutos de ambas magnitudes. Esto se ha hecho con la intención de comparar ambas. En la figura 8 se presentan las estadísticas asociadas al nivel de agregación de provincias y comunidad autónoma (están realizadas del mismo modo para todas las comarcas y municipios) por porcentajes y la relación entre ambos, con un formato que nos avisa por una parte sobre la presencia visual (cuanto es percibida según el parámetro de Accesibilidad Visual Ponderada , AVP, $\mathrm{h}=0$, psi) de cada clase o cobertura de suelo estudiada respecto a las demás en ese nivel de agregación (si sumamos los elementos de cada fila obtenemos el $100 \%$ de la cobertura); por otra parte nos informa de su presencia superficial (la de cada clase respecto al total de la superficie). Por último se presenta en la parte inferior de la figura 7 la relación entre ambos porcentajes, con un código de colores igualmente relacionado con su valor que avisa de la preponderancia visual de una cobertura respecto a su superficie. Valores más cálidos (desde el 
Romero Romero, D., Romero Morato, A, Guerrero Álvarez, J.J., Giménez de Azcarate Fernández, F., Cáceres Clavero, F., Moreira Madueño, J.M. (2016): "Análisis comparativo entre la distribución de usos del suelo y su accesibilidad visual”, GeoFocus (Artículos), no 20, p. 63-85. ISSN: 1578-5157 http://dx.doi.org/10.21138/GF.516

amarillo hasta el rojo) nos avisan que una clase es poco percibida visualmente, o tiene poca presencia visual respecto a la superficie que ocupa. Valores más fríos (desde el amarillo al verde), nos avisan de coberturas o clases de suelo muy visibles respecto a su superficie (normalmente están ligadas a determinadas categorías de suelo sellado).

\begin{tabular}{|c|c|c|c|c|c|c|c|c|c|c|c|c|c|c|c|c|c|}
\hline \\
\hline \multirow[b]{3}{*}{ entidad } & \multicolumn{7}{|c|}{ forestal } & \multicolumn{5}{|c|}{ agrícola } & \multicolumn{5}{|c|}{ sellado } \\
\hline & \multicolumn{4}{|c|}{ arbolado } & \multirow[b]{2}{*}{$\mathrm{ma}$} & \multirow[b]{2}{*}{ he } & \multirow[b]{2}{*}{ su } & \multirow[b]{2}{*}{ ch } & \multicolumn{4}{|c|}{ leñoso } & \multirow[b]{2}{*}{ la } & \multirow[b]{2}{*}{ ed } & \multirow[b]{2}{*}{$\mathrm{vl}$} & \multirow[b]{2}{*}{$\mathrm{zV}$} & \multirow[b]{2}{*}{ ze } \\
\hline & qu & co & eu & of & & & & & $\mathrm{ci}$ & ol & vi & oc & & & & & \\
\hline Almería & 1,52 & 4,58 & 0,19 & 0,34 & 25,15 & 22,04 & 20,63 & 11,74 & 0,81 & 1,11 & 0,19 & 3,70 & 0,48 & 3,21 & 3,64 & 0,39 & 0,30 \\
\hline Cádiz & 5,08 & 3,17 & 0,56 & 2,19 & & 16,67 & 6,82 & & 0,21 & 2,27 & 1,90 & 0,46 & 2,39 & 5,85 & 9,78 & 2,84 & 0,25 \\
\hline Córdoba & 4,48 & 1,22 & 0,05 & 1,38 & 10,29 & 9,84 & 5,90 & 26,13 & 1,05 & 27,11 & 0,92 & 0,58 & 0,63 & 4,56 & 4,53 & 0,94 & 0,39 \\
\hline Granada & 1,99 & 6,87 & 0,01 & 0,93 & 16,69 & 14,88 & 11,62 & 15,09 & 0,08 & 11,98 & 0,33 & 7,99 & 0,36 & 4,86 & 5,07 & 0,78 & 0,47 \\
\hline Huelva & 6,30 & 5,18 & 1,92 & 0,73 & 16,25 & 15,65 & 10,81 & 15,96 & 2,23 & 5,32 & 0,90 & 2,08 & 3,68 & 5,61 & 5,08 & 1,32 & 0,99 \\
\hline Jaén & 3,77 & 6,82 & 0,01 & 0,46 & 11,88 & 12,01 & 8,96 & 4,78 & 0,00 & 44,12 & 0,05 & 0,70 & 0,53 & 2,71 & 2,73 & 0,24 & 0,23 \\
\hline Málaga & 2,27 & 5,22 & 0,08 & 0,99 & 19,99 & 19,77 & 9,01 & 8,15 & 1,45 & 11,01 & 0,38 & 3,96 & 0,52 & 6,64 & 7,46 & 2,72 & 0,37 \\
\hline Sevilla & 2,10 & 0,48 & 0,24 & 0,60 & 4,84 & 6,45 & 6,19 & 38,83 & 2,74 & 17,21 & 0,14 & 1,14 & 0,63 & 6,46 & 10,00 & 1,23 & 0,72 \\
\hline \multirow[t]{2}{*}{ ANDALUCIA } & 3,07 & 4,38 & 0,24 & 0,97 & 15,52 & 14,97 & 10,17 & 17,49 & 0,93 & 14,89 & 0,55 & 3,08 & 0,90 & 4,99 & 6,10 & 1,33 & $\overline{0,42}$ \\
\hline & & & & & & & 49,31 & & & & & 36,94 & 0,90 & & & & 12,84 \\
\hline
\end{tabular}

PORCENTAJE TERRITORIO

\begin{tabular}{|c|c|c|c|c|c|c|c|c|c|c|c|c|c|c|c|c|c|}
\hline \multirow[b]{3}{*}{ entidad } & \multicolumn{7}{|c|}{ forestal } & \multicolumn{5}{|c|}{ agrícola } & \multicolumn{5}{|c|}{ sellado } \\
\hline & \multicolumn{4}{|c|}{ arbolado } & \multirow[b]{2}{*}{$\mathrm{ma}$} & \multirow[b]{2}{*}{ he } & \multirow[b]{2}{*}{ su } & \multirow[b]{2}{*}{ ch } & \multicolumn{4}{|c|}{ leñoso } & \multirow[b]{2}{*}{ la } & \multirow[b]{2}{*}{ ed } & \multirow[b]{2}{*}{$\mathrm{vl}$} & \multirow[b]{2}{*}{ zv } & \multirow[b]{2}{*}{ ze } \\
\hline & qu & co & eu & of & & & & & $\mathrm{ci}$ & ol & vi & oc & & & & & \\
\hline Almería & 1,63 & 6,03 & 0,14 & 0,36 & 24,27 & 21,84 & 18,78 & 11,81 & 1,07 & 1,88 & 0,20 & 7,74 & 0,30 & 1,13 & 2,41 & 0,13 & 0,28 \\
\hline Cádiz & 8,73 & 1,54 & 0,39 & 3,23 & 14,97 & 18,08 & 4,31 & 34,90 & 0,31 & 2,80 & 1,48 & 0,34 & 2,40 & 1,74 & 3,84 & 0,72 & 0,22 \\
\hline Córdoba & 10,09 & 2,59 & 0,07 & 0,54 & 12,44 & 16,66 & 5,73 & 23,16 & 0,69 & 22,90 & 0,52 & 0,36 & 1,00 & 0,92 & 1,95 & 0,16 & 0,23 \\
\hline Granada & 2,83 & 6,07 & 0,01 & 0,82 & 17,48 & 15,83 & 11,81 & 19,23 & 0,06 & 14,01 & 0,27 & 7,76 & 0,46 & 0,96 & 2,05 & 0,11 & 0,24 \\
\hline Huelva & 8,57 & 5,86 & 4,02 & 0,75 & 23,64 & 24,58 & 11,74 & 8,95 & 1,68 & 3,22 & 0,54 & 1,02 & 1,94 & 0,87 & 1,85 & 0,23 & 0,53 \\
\hline Jaén & 4,70 & 7,04 & 0,02 & 0,59 & 15,21 & 14,53 & 8,51 & 5,38 & 0,00 & 39,87 & 0,03 & 0,66 & 0,95 & 0,70 & 1,58 & 0,07 & 0,14 \\
\hline Málaga & 4,51 & 4,45 & 0,11 & 1,21 & 18,83 & 17,34 & 6,89 & 14,06 & 1,68 & 17,25 & 0,56 & 5,20 & 0,73 & 2,38 & 3,68 & 0,81 & 0,31 \\
\hline Sevilla & 6,03 & 0,67 & 0,40 & 0,58 & 9,81 & 12,25 & 5,26 & 40,91 & 1,74 & 14,35 & 0,11 & 0,81 & 1,66 & 1,78 & 3,06 & 0,27 & 0,33 \\
\hline \multirow[t]{2}{*}{ ANDALUCIA } & 5,96 & 4,26 & 0,60 & 0,88 & 16,40 & 17,12 & 8,94 & 20,16 & 0,86 & 16,30 & 0,40 & 2,76 & 1,15 & 1,24 & 2,43 & 0,26 & 0,28 \\
\hline & & & & & & & 54,16 & & & & & 40,49 & 1,15 & & & & 4,20 \\
\hline
\end{tabular}

PORCENTAJE VISIBILIDAD/ PORCENTAJE TERRITORIO

\begin{tabular}{|c|c|c|c|c|c|c|c|c|c|c|c|c|c|c|c|c|c|}
\hline \multirow[b]{3}{*}{ entidad } & \multicolumn{7}{|c|}{ forestal } & \multicolumn{5}{|c|}{ agrícola } & \multicolumn{5}{|c|}{ sellado } \\
\hline & \multicolumn{4}{|c|}{ arbolado } & \multirow[b]{2}{*}{$\mathrm{ma}$} & \multirow[b]{2}{*}{ he } & \multirow[b]{2}{*}{ su } & \multirow[b]{2}{*}{ ch } & \multicolumn{4}{|c|}{ leñoso } & \multirow[b]{2}{*}{ la } & \multirow[b]{2}{*}{ ed } & \multirow[b]{2}{*}{$\mathrm{vl}$} & \multirow[b]{2}{*}{ zV } & \multirow[b]{2}{*}{ ze } \\
\hline & qu & co & eu & of & & & & & $\mathrm{ci}$ & ol & vi & oc & & & & & \\
\hline Almería & 0,93 & 0,76 & 1,36 & 0,95 & 1,04 & 1,01 & 1,10 & 0,99 & 0,76 & 0,59 & 0,92 & 0,48 & 1,61 & 2,83 & 1,51 & 3,14 & 1,08 \\
\hline Cádiz &, 58 & 2,06 & 1,44 & 0,68 & 1,05 & 0,92 & 1,58 & 68 & 0,70 & 81 & 1,28 & 1,35 & 1,00 & 36 & 55 & 3,94 & 1,15 \\
\hline Córdoba & 0,44 & 0,47 & 0,84 & 2,57 & 0,83 & 0,59 & 1,03 & 1,13 & 1,51 & 1,18 & 1,79 & 1,59 & 0,63 & 4,97 & 2,32 & 5,86 & 1,70 \\
\hline Granada & 0,70 & 1,13 & 1,44 & 1,14 & 0,95 & 0,94 & 0,98 & 0,78 & 1,32 & 0,85 & 1,23 & 1,03 & 0,77 & 5,05 & 2,47 & 6,97 & 1,99 \\
\hline Huelv & 0,74 & 0,88 & 0,48 & 0,97 & 0,69 & 0,64 & 0,92 & 1,78 & 1,32 & 1,65 & 1,65 & 2,05 & 1,90 & 6,44 & 2,75 & 5,85 & 1,86 \\
\hline Jaén & 0,80 & 0,97 & 0,70 & 0,77 & 0,78 & 0,83 & 1,05 & 0,89 & 1,18 & 1,11 & 1,65 & 1,05 & 0,56 & 3,84 & 1,73 & 3,44 & 1,60 \\
\hline Málaga & 0,50 & 1,17 & 0,72 & 0,83 & 1,06 & 1,14 & 1,31 & 0,58 & 0,87 & 0,64 & 0,68 & 0,76 & 0,72 & 2,79 & 2,03 & 3,36 & 1,20 \\
\hline Sevilla & 0,35 & 0,72 & 0,59 & 1,04 & 0,49 & 0,53 & 1,18 & 0,95 & 1,58 & 1,20 & 1,29 & 1,41 & 0,38 & 3,63 & 3,27 & 4,58 & 2,18 \\
\hline ANDALUCIA & 0,51 & 1,03 & 0,40 & 1,09 & 0,95 & 0,87 & 1,14 & 0,87 & 1,08 & 0,91 & 1,39 & 1,11 & 0,78 & 4,04 & 2,51 & 5,06 & 1,52 \\
\hline
\end{tabular}

Figura 8. Datos estadísticos sobre percepción visual y ocupación del suelo.

Fuente de elaboración propia. 
Romero Romero, D., Romero Morato, A, Guerrero Álvarez, J.J., Giménez de Azcarate Fernández, F., Cáceres Clavero, F., Moreira Madueño, J.M. (2016): "Análisis comparativo entre la distribución de usos del suelo y su accesibilidad visual”, GeoFocus (Artículos), no 20, p. 63-85. ISSN: 1578-5157 http://dx.doi.org/10.21138/GF.516

\subsection{Análisis de los resultados}

Un somero análisis a nivel provincial y autonómico sobre los resultados arrojados en las tablas de la figura 8 nos permite verificar algunas cuestiones que a priori nos parecen claras, o que damos por sentadas como es la presencia del olivar en la provincia de Jaén, y en menor medida en Córdoba y Sevilla; así como las grandes extensiones de cultivos herbáceos en la provincia de Sevilla y Cádiz y en menor medida Córdoba y Granada, asociadas a sus vegas y campiñas ; pero podemos comprobar que en algunos casos estas extensiones de cultivo pueden ser muy visibles (caso de olivar de Jaén). Podemos detectar por el código de colores referido, que existe en todas las provincias (quizá a excepción de Sevilla) una gran superficie forestal de matorral, herbáceo y suelo desnudo

Del mismo modo y a nivel autonómico, es fácil deducir que la edificación es altamente percibida visualmente respecto a la superficie que ocupa. Ello es debido a que los núcleos urbanos son conspicuos y visibles desde categorías de suelo en las que es altamente probable encontrar observadores. También arroja valores que nos permiten realizar lecturas sobre formaciones como las dehesas, en las que las quercíneas - forestal (Sup. $=6 \%$, Vis. $=3,1 \%$ ), son poco visibles respecto a la superficie que ocupan, seguramente debido a la estructura de la propiedad (conformada por grandes extensiones lejanas a núcleos poblados y vías de comunicación, en las que es poco probable encontrar observadores).

A nivel comarcal, entidades que abarcan realidades territoriales diversas como el Valle Medio del Guadalquivir (Piedemonte de Sierra Morena, Vega Alta del Guadalquivir y Campiña de Sevilla y Córdoba) ofrecen lecturas como las asociadas a la visibilidad de los cultivos herbáceos (muy por encima de su superficie real). Esto es explicable por el paso de las vías de comunicación paralelas al Guadalquivir y a la visibilidad que proporciona de estos cultivos las localizaciones más elevadas (Sierra Morena). Comprobamos también que todas las categorías asociadas a suelos forestales tienen poca visibilidad respecto al porcentaje de superficie que ocupan (cosa lógica si comprobamos la proporción que en la misma comarca ocupa el Parque Natural de la Sierra de Hornachuelos).

Este análisis, cuando se va haciendo más local nos habla de particularidades del ámbito territorial seleccionado. Así a nivel provincial, y tomando como ejemplo Huelva deducimos de la presencia (singular respecto a otras provincias) de eucaliptos en zonas poco visibles, la actividad de ENCE (Energía y Celulosa) y su relación con la gestión de espacios forestales.

A nivel de término municipal, existen casos como el de Andújar, en el que la percepción visual de las coberturas correspondientes a Olivar supera con creces la realidad existente, es decir que debido a la situación geográfica de determinadas categorías de accesibilidad local, podemos tener una percepción equivocada de la realidad, o que no se corresponde con la extensión superficial de dichos cultivos. En el caso que nos ocupa seguramente sea debido a que dichos cultivos se encuentran en entornos de vías principales que atraviesan el término, como la A-92.

\subsection{El visor de comparación de estadísticas}

Los resultados pueden ser visualizados conjuntamente y de forma comparada gracias al diseño y programación de una página web para la difusión de los datos estadísticos almacenados. La 
Romero Romero, D., Romero Morato, A, Guerrero Álvarez, J.J., Giménez de Azcarate Fernández, F., Cáceres Clavero, F., Moreira Madueño, J.M. (2016): "Análisis comparativo entre la distribución de usos del suelo y su accesibilidad visual”, GeoFocus (Artículos), no 20, p. 63-85. ISSN: 1578-5157 http://dx.doi.org/10.21138/GF.516

navegación interactiva entre los distintos ámbitos geográficos a través del cambio entre distintos niveles de zoom y simultáneamente sobre la comparativa de estadísticas, permite responder a distintos niveles de precisión temática. Todo ello con criterio de máxima simplicidad y claridad (figura 9).

En la aplicación que se ha desarrollado en el seno de este trabajo, la navegación geográfica se ciñe a 3 niveles de desagregación territorial, correspondientes con cada uno de los ámbitos de gestión definidos. Es posible activar cualquier delimitación y obtener un mapa más detallado con sus subdivisiones.

Los datos geográficos referidos a cada nivel de zoom, para la entidad seleccionada en cuestión, quedan disponibles, no siendo accesibles los datos relativos a otros niveles de zoom o entidades geográficas no representadas. Pulsando sobre el icono de gráfica se pueden obtener los datos y la gráfica comparativa para la división administrativa activa en cada momento (figura 9).

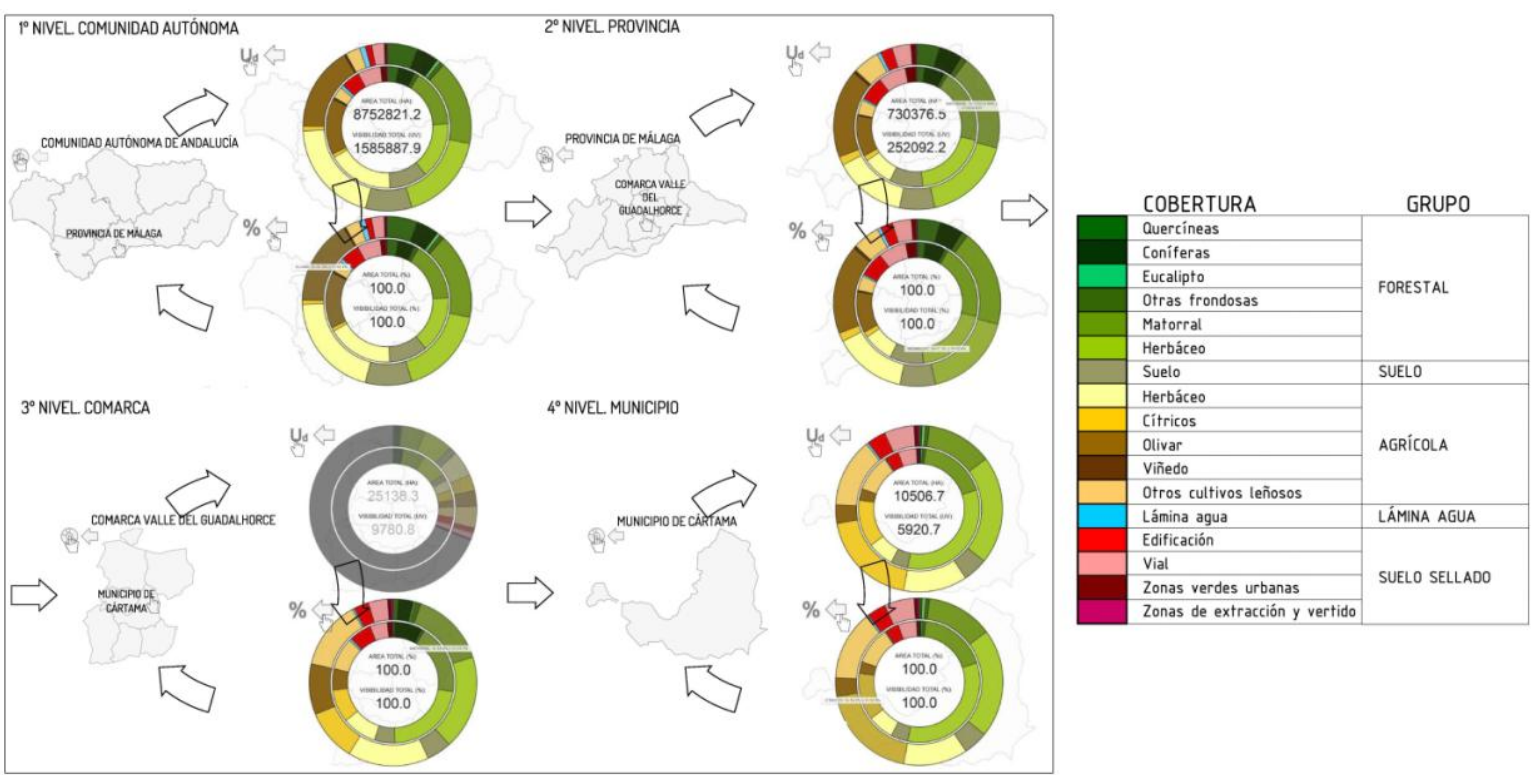

Figura 9. Ejemplo de navegación a través del visor comparador de estadísticas.

Fuente de elaboración propia sobre datos de la REDIAM

Por otro lado, y para cualquier entidad seleccionada además de lo anteriormente descrito, dispondremos de dos cifras globales, la accesibilidad visual ponderada en UV (unidades de visibilidad) total para la entidad seleccionada y la superficie total en hectáreas de dicha entidad. Por otra parte y con la gráfica activa, se puede pasar de unidades absolutas a porcentajes pulsando sobre el icono de 'cambiar unidad'. Esto cambiará también el contenido de las etiquetas emergentes (figura 9).

Los datos temáticos quedan cargados para cada entidad geográfica seleccionada, que sólo podrá ser una, y quedarán presentados mediante una gráfica sencilla, en la que se reflejarán dos datos. En la gráfica, la corona exterior representa la proporción real de cada cobertura, mientras que la interior representa la incidencia visual de las mismas. Las 17 coberturas básicas evaluadas se visualizan siempre en el mismo orden, y pueden ser agrupadas fácilmente. 
Romero Romero, D., Romero Morato, A, Guerrero Álvarez, J.J., Giménez de Azcarate Fernández, F., Cáceres Clavero, F., Moreira Madueño, J.M. (2016): "Análisis comparativo entre la distribución de usos del suelo y su accesibilidad visual”, GeoFocus (Artículos), no 20, p. 63-85. ISSN: 1578-5157 http://dx.doi.org/10.21138/GF.516

Este visor, elaborado por el equipo de paisaje de la REDIAM, está disponible en el apartado de 'herramientas de difusión', en la web del Laboratorio REDIAM:

(http://laboratoriorediam.cica.es/estusossuelo/index.html)

\section{Discusión}

Con las magnitudes aportadas por la Accesibilidad Visual, no se realiza valoración cualitativa alguna respecto al paisaje o a la manifestación visual de este, más allá de la que se puede deducir de la consideración de determinados efectos que hacen difusa las distintas cuencas visuales asociadas a cada punto de observación, como la distancia y proyección visual (Romero et al., 2014). En el caso de SVA se calcularon parámetros y magnitudes según una malla regular de $100 \mathrm{~m}$ x100m (más de 11 millones de localizaciones), un supuesto que partía de la continuidad en las propiedades visuales en el área de cada tesela de 1 hectárea de superficie. Su resultado se traduce en la disposición de una imagen de $360^{\circ}$ por cada hectárea de territorio, conformada por las líneas de ruptura del horizonte (Romero et al., 2016).

El tratamiento cumulativo de las cuencas visuales (Tabik et al., 2012) nos permite estimar los lugares (trasladados a píxeles) observados con mayor frecuencia, considerando la heterogeneidad en la distribución de espectadores que estimemos oportuna (Guerrero et al., 2010). $\mathrm{Su}$ aplicación viene siendo útil en estudios que explican determinados aspectos estructurales de un paisaje (Llobera et al., 2010); además el número de localizaciones de observación distribuidas uniformemente es proporcional a la respuesta proporcionada por el territorio en términos de visibilidad. En el caso de SVA se calcularon según una malla regular de 100m x100m (más de 11 millones), un punto de partida que presuponía a estos efectos, la continuidad en las propiedades visuales estudiadas en el área de esta tesela.

Los aspectos relacionados con la percepción visual del territorio vienen acarreando diversidad de opiniones en cuanto al planteamiento, al método para valorarla y la aplicabilidad de estos en las tareas de gestión territorial. Mediante la comparativa propuesta con la ocupación real del suelo en cuanto a coberturas podemos deducir no sólo las razones que pueden hacer más visible a un territorio o una porción del mismo (localización de infraestructuras viarias, poblaciones...lugares en definitiva muy frecuentados en la hipótesis tentativa de distribución de espectadores). Al disponer de otra relación cuantitativa, esta vez sobre "cuánto ocupa" lo que se ve (su realidad biófisica) y "cuánto se ve", es posible trabajar estableciendo criterios espaciales que ayuden a fomentar o a preservar determinadas imágenes del territorio.

El análisis espacial de la visibilidad en términos de intervisibilidad, aplicada a su relación con los usos de suelo y sus coberturas se ha testeado para la monitorización de sus cambios en intervalos periódicos usando metodologías similares (Miller et al. 2001 y Miller y Law, 1997). Su utilidad puede ser complementaria con técnicas demoscópicas de evaluación de la calidad visual.

Algunas de ellas se basan en encuestas directas sobre muestras de población en torno determinadas cuestiones de preferencia y conocimiento (Ecobarómetro de Andalucía, 2011); otras, 
Romero Romero, D., Romero Morato, A, Guerrero Álvarez, J.J., Giménez de Azcarate Fernández, F., Cáceres Clavero, F., Moreira Madueño, J.M. (2016): "Análisis comparativo entre la distribución de usos del suelo y su accesibilidad visual”, GeoFocus (Artículos), no 20, p. 63-85. ISSN: 1578-5157 http://dx.doi.org/10.21138/GF.516

combinan estos aspectos con otros parámetros en base a la ocupación del suelo (Corine Land Cover, CLC) y algunas características inherentes a la orografía, como la pendiente y la orientación (Arriaza et al., 2004).

En otras ocasiones las encuestas se focalizaron en perfilar valores escénicos en base a indicadores paisajísticos elaborados como índices de diversidad y heterogeneidad (Dramstad et al., 2006, Roth \& Gruehn, 2006, Frank et al., 2013, Ode et al., 2009); o en la elaboración de índices cualitativos en base a la toma de datos en el terreno (Palmieri et al., 2001) y a la evolución de la complejidad y la fragmentación de los usos del suelo (Herzog y Lausch, 2000). En cualquiera de los casos expuestos cualquier valoración de la calidad del paisaje puede parecer contradictoria con el citado artículo 1 del Convenio Europeo.

La base sobre ocupación de suelo SIOSE-SIPNA viene sirviendo ya desde la REDIAM como referencia para trabajos relacionados con el paisaje. Uno de ellos parte de la correspondencia de la misma con la delimitación de ámbitos paisajísticos y unidades fisionómicas, a partir de las cuales se extrajo información sintética condensada en tres indicadores: naturalidad, riqueza y diversidad paisajística. Las continuas actualizaciones de las bases de referencia han propiciado el análisis de estos parámetros desde un punto de vista evolutivo, pudiéndose valorar los cambios ocurridos en las unidades fisionómicas consideradas para los tres índices mencionados entre los años 2009 y 2011 (IMA, 2015). En este sentido no sería difícil inferir la relación entre dichos indicadores y la Accesibilidad Visual del Territorio.

\section{Conclusiones}

\subsection{Sobre la metodología.}

Los 17 rasters obtenidos han sido utilizados al objeto de ser combinados con los rasters de accesibilidad visual para obtener los datos estadísticos sobre cuáles son los usos del suelo que caracterizan visualmente al territorio, pero además:

- El procedimiento de generación de los rasters pone de manifiesto de una forma muy sencilla errores semánticos y geométricos en la capa de origen SIOSE OS. Por ejemplo, se han detectado 'overlaps' de polígonos o contenidos que no suponen el $100 \%$ de la cobertura. Además, permite la visualización ágil de la capa al completo, incluso con transparencia y orto debajo, gracias a las pirámides, con lo que la detección visual de errores puede resultar más sencilla.

- Podrían ser aplicados a la obtención sencilla de combinaciones entre cobertura del suelo y cualquier set de datos ráster, como pueden ser los datos sobre clima (por ejemplo, para estudiar cuáles son los ámbitos climáticos donde se dan determinadas coberturas).

- Del mismo modo puede ser combinado con otros ámbitos geográficos específicos. Algunos que pueden ser útiles son los asociados a la Red de Espacios Protegidos de Andalucía (RENPA), o a la delimitación de unidades visuales homogéneas o cuencas visuales cuya conformación no depende de un punto de observación sino de todos los posibles a la vez (Alcántara et al., 2014). 
Romero Romero, D., Romero Morato, A, Guerrero Álvarez, J.J., Giménez de Azcarate Fernández, F., Cáceres Clavero, F., Moreira Madueño, J.M. (2016): "Análisis comparativo entre la distribución de usos del suelo y su accesibilidad visual”, GeoFocus (Artículos), no 20, p. 63-85. ISSN: 1578-5157 http://dx.doi.org/10.21138/GF.516

\subsection{Sobre los resultados.}

La accesibilidad visual de un territorio no se corresponde en muchos casos con la realidad territorial. Podemos tener la consideración de que en un determinado municipio existe mucha superficie dedicada a un uso concreto, cuando en realidad ésta es escasa. Ello depende en gran medida de los lugares desde los cuales se observa y como son de accesibles.

Así, lugares con más posibilidad de ser frecuentados (según su accesibilidad visual) pueden originar mayor influencia en la percepción global o en la imagen que se tiene del mismo; es decir los terrenos visibles desde carreteras se hacen observables por mucha gente, a pesar de que ocupen poca extensión, dado que son lugares muy transitados. Opuestamente lugares poco transitados pueden contener vistas singulares, pero éstas se estiman no tienen tanta influencia en el imaginario colectivo porque poca gente los frecuenta.

Por otra parte, para cualquier lugar desde el que observemos, dado que el territorio tiene relieve (y por encima de éste, árboles, edificaciones etc.), podemos decir que hay mucho o poco 'territorio visible'. Ambas cuestiones influyen en la configuración del imaginario de nuestro entorno: la generalizada imagen de Andalucía como territorio calmo y seco está en parte provocada por que las vías de comunicación principales transcurren por los distintos valles, dejando en un lejano segundo plano los territorios más húmedos y montañosos. La construcción del paso del AVE ha cambiado parcialmente este concepto, puesto que atravesando los Pedroches se observa un paisaje más verde y abrupto.

Si bien con la aplicación propuesta es posible comparar datos asociados a determinados aspectos mensurables de la visibilidad del territorio según diversas unidades administrativas, en la misma aún se echa de menos visualizar la conformación de esas unidades en cuanto a su relieve; o incluso la traslación del diagrama referente a su piel, al mapa representado. Pero los fines para los que se ha diseñado (de comparación estadística pura), y sobre todo la rapidez en la salida gráfica ha condicionado la decisión hacia el resultado propuesto.

\section{Referencias bibliográficas}

Aguiló, M. (1981): Metodología para la evaluación de la fragilidad visual del paisaje. Tesis doctoral. E.T.S. Ing. de Caminos, Univ. Politécnica de Madrid, 1981.

Arriaza, M., Cañas, J. F., Cañas, J. A., y Ruiz, P. (2004): “Assessing the visual quality of rural landscapes", Landscape and urban planning, 69(1), 115-125.

Alcántara, J., Romero, D., Romero, A., Ghislanzoni, M., Guerrero, J.J., Giménez de Azcárate, F., Cáceres, F., Moreira, J.M. (2014): "Obtención de horizontes visuales a partir del Sistema de Visibilidad de Andalucía (REDIAM) y su aplicación en la identificación de unidades de paisaje", en Ramón, A. (Ed.), Actas del XVI Congreso Nacional de Tecnologías de la Información Geográfica. Publicación de acceso abierto (Open Access) bajo la licencia CC BY-NC-ND. http://creativecommons.org/licenses/by-nc-nd/4.0/, 302-312. Junio 2014, Alicante. XVI Congreso Nacional de Tecnologías de la Información Geográfica. 
Romero Romero, D., Romero Morato, A, Guerrero Álvarez, J.J., Giménez de Azcarate Fernández, F., Cáceres Clavero, F., Moreira Madueño, J.M. (2016): "Análisis comparativo entre la distribución de usos del suelo y su accesibilidad visual”, GeoFocus (Artículos), no 20, p. 63-85. ISSN: 1578-5157 http://dx.doi.org/10.21138/GF.516

Aramburu, M., Cifuentes p., Escribano, R., y González, S. (1994): Guía para la elaboración de estudios del medio físico. Contenido y metodología. Ministerio de Obras Públicas y Transporte. Secretaría del Estado para las políticas de Agua y Medio Ambiente. Madrid, 809p.

Berry, J. K. (1993): “Cartographic Modeling: The Analitycal Capabilities of GIS”, en Goodchild, M. F.; Parks, B.O. y Steyaert, L.T. (eds.): Environmental Modeling with GIS, Oxford University Press, Nueva York, 58-74.

Cáceres, F., Romero, D., Guerrero, J.J., Ghislanzoni, M., Giménez de Azcárate, F., Moreira, J.M. (2014). "Parametrización numérica de las relaciones visuales del territorio: el Sistema de Visibilidad de Andalucía. REDIAM", en Ramón, A. (Ed.), Actas del XVI Congreso Nacional de Tecnologías de la Información Geográfica. Publicación de acceso abierto (Open Access) bajo la licencia CC BY-NC-ND. http://creativecommons.org/licenses/by-nc-nd/4.0/, 418-430. Junio 2014, Alicante. XVI Congreso Nacional de Tecnologías de la Información Geográfica.

Consejo de Europa (2000): "Convenio Europeo del Paisaje". Convención Europea del Paisaje. Octubre 2000. Florencia.

Dramstad, W. E., Tveit, M. S., Fjellstad, W. J., y Fry, G. L. (2006): "Relationships between visual landscape preferences and map-based indicators of landscape structure", Landscape and urban planning, 78(4), 465-474.

Domingo-Santos, J.M., Fernández de Villarán, R., Rapp-Arrarás, I., Corral-Pazos de Provens, E. (2011): "The visual exposure in forest and rural landscapes: An algorithm and a GIS tool", Landscape and Urban Planning 101, 52-58.

Fisher, P.F. (1992): "First Experiments in Viewshed Uncertainty: Simulating Fuzzy Viewsheds", Photogrammetric Engineering \& Remote. Sensing, 58, 3, 345-352.

Fisher, P.F. (1996): "Extending the Applicability of Viewsheds in Landscape Planning". Photogrammetric Engineering \& Remote. Sensing, 62, 11, 1297-1302.

Frank, S., Fürst, C., Koschke, L., Witt, A., y Makeschin, F. (2013): “Assessment of landscape aesthetics - validation of a landscape metrics-based assessment by visual estimation of the scenic beauty", Ecological Indicators, 32, 222-231.

Gil, Y., Romero, D., Ortega, E., Domínguez, M.C., Navas, P., Patiño, M., Vicent, C., Santos, M., Quijada, J., Giménez de Azcárate, F., Cáceres, F. y Moreira, J.M. (2010): "SIOSE Andalucía, experiencia de integración y actualización de bases cartográficas multiescala", en: Ojeda, J., Pita, M.F. y Vallejo, I. (Eds.), Tecnologías de la Información Geográfica: La Información Geográfica al servicio de los ciudadanos. Sevilla, Secretariado de Publicaciones de la Universidad de Sevilla, 116-134. Septiembre 2010, Sevilla. XIV Congreso Nacional de Tecnologías de la Información Geográfica.

Goodchild M. y Haining, R., (2005): "SIG y análisis espacial de datos: perspectivas convergentes" Investigaciones Regionales, 6, 2005, 175-201.

Guerrero, J.J., Ghislanzoni, M., Romero, D., Cáceres, F., Giménez de Azcarate, F. y Moreira, J.M. (2010): "Identificación y caracterización del Paisaje mediante parámetros visuales del relieve. REDIAM", en: Ojeda, J., Pita, M.F. y Vallejo, I. (Eds.), Tecnologías de la Información Geográfica: La Información Geográfica al servicio de los ciudadanos. Sevilla, Secretariado de Publicaciones de 
Romero Romero, D., Romero Morato, A, Guerrero Álvarez, J.J., Giménez de Azcarate Fernández, F., Cáceres Clavero, F., Moreira Madueño, J.M. (2016): "Análisis comparativo entre la distribución de usos del suelo y su accesibilidad visual”, GeoFocus (Artículos), no 20, p. 63-85. ISSN: 1578-5157 http://dx.doi.org/10.21138/GF.516

la Universidad de Sevilla, 841-860. Septiembre 2010, Sevilla. XIV Congreso Nacional de Tecnologías de la Información Geográfica.

Herzog, F. y Lausch, A. (2001): "Supplementing land-use statistics with landscape metrics: some methodological considerations", Environmental Monitoring and Assessment 72, 37-50.

Higashiyama, A., Shimono, K. (1984): "How accurate is size and distance perception for very far terrestrial objects", Perception \& Psychophysics 55, 429-442.

Miller, D. (2001): “A method for estimating changes in the visibility of land cover”. Landscape and Urban Planning, 54 (1), 93-106.

Miller, D. y Law, A.(1997): "The Mapping of Terrain Visibility". The Cartographic Journal, 34 (2), 87-91.

Mérida, M., (1996): "El Paisaje Visual”. Baetica. Estudios de Arte. Geografía e Historia, 18, 205222.

Llobera, M., (2003): "Extending GIS-based visual analysis: the concept of visualscapes". Int. J. Geographical Information Science, 17, 1, 25-48.

Llobera, M; Wheatley, D., Steele, J; Cox, S., Parchment, O. (2010): "Calculating the inherent visual structure of a landscape (inherent viewshed) using high-throughput computing". Beyond the artefact: Digital Interpretation of the Past: Proceedings of CAA2004, 146-151. Abril 2004, Prato.

Ode, Å., Fry, G., Tveit, M. S., Messager, P., y Miller, D. (2009): "Indicators of perceived naturalness as drivers of landscape preference", Journal of environmental management, 90(1), 375383.

Olaya V., (2011): "Sistemas de Información Geográfica", http://volaya.github.io/libro-sig/ (consultado 13/09/2017).

Palmieri, A., Dominici, P., Kasanko, M.,y Martino, L. (2011): "Diversified landscape structure in the EU Member States Landscape indicators from the LUCAS 2009 survey". Eurostat. Statistics in focus 21/2011. http://ec.europa.eu/eurostat/documents/3433488/5578816/KS-SF-11-021EN.PDF/ad633632-9a74-4cc1-9834-b647699e3f58 (consultado 13/09/2017).

Romero, D., Guerrero, J.J., Ghislanzoni, M., Romero, A., Sillero Almazán, J.M., Giménez de Azcárate, F., Cáceres, F., Moreira, J.M. (2014): "Evaluación de la accesibilidad visual del territorio ante distintas intervenciones a través de la explotación del sistema de visibilidad de Andalucía. REDIAM", en Ramón, A. (Ed.), Actas del XVI Congreso Nacional de Tecnologías de la Información Geográfica. Publicación de acceso abierto (Open Access) bajo la licencia CC BY-NCND. http://creativecommons.org/licenses/by-nc-nd/4.0/. 226-239. Junio 2014, Alicante. XVI Congreso Nacional de Tecnologías de la Información Geográfica.

Romero, D., Romero, A. L., Guerrero, J. J., Montes, L. C., de Giménez de Azcárate, F., Cáceres, F., y Moreira, J. M. (2016): “Análisis comparativo entre la distribución de usos del suelo en Andalucía y su percepción visual. REDIAM", en Galacho, F.B., Vías, J., y Reyes, S. (Eds.) Actas del XVII Congreso Nacional de Tecnologías de Información Geográfica, Aplicaciones de las Tecnologías de la Información Geográfica (TIG) para el desarrollo económico sostenible. 274-285, 29, 30 de junio y 1 de julio 2016, Málaga. XVII Congreso Nacional de Tecnologías de la Información Geográfica. 
Romero Romero, D., Romero Morato, A, Guerrero Álvarez, J.J., Giménez de Azcarate Fernández, F., Cáceres Clavero, F., Moreira Madueño, J.M. (2016): "Análisis comparativo entre la distribución de usos del suelo y su accesibilidad visual”, GeoFocus (Artículos), no 20, p. 63-85. ISSN: 1578-5157 http://dx.doi.org/10.21138/GF.516

Romero, D., Romero, A. L., Guerrero, J. J., Montes, L. C., de Giménez de Azcárate, F., Cáceres, F., y Moreira, J. M. (2016): "Realidad esquematizada. Interpretación del paisaje a través de sus horizontes visuales. REDIAM", en Galacho, F.B., Vías, J., y Reyes, S. (Eds.) Actas del XVII Congreso Nacional de Tecnologías de Información Geográfica, Aplicaciones de las Tecnologías de la Información Geográfica (TIG) para el desarrollo económico sostenible. 298-308, 29, 30 de junio y 1 de julio 2016, Málaga. XVII Congreso Nacional de Tecnologías de la Información Geográfica.

Roth, M., Gruehn, D., (2012): "Visual landscape assessment for large areas - using GIS, internet surveys and statistical methodologies in participatory landscape planning for the Federal State of Mecklenburg-Western Pomerania”, Germany. Latvian Acad. Sci. A: Humanit. Soc. Sci., 2012: 129142.

Tabik, S., Romero, L.F., y Zapata, E.L., (2012): "Simultaneous computation of total viewshed on large high resolution grids". International Journal of Geographical Information Science, 27 (4), 804-814.

Tomlin, C. D. (1990): Geographic information systems and cartographic modeling. Prentice Hall, Englewood Cliffs (NJ). 572 p.

Tomlin, C.D. (1994): "Map Algebra. One perspective”. Landscape and Urban Planning 30, 3-12.

Wheatley, D. (1995): "Cumulative viewshed analysis, a GIS-based method for investigating intervisibility, and its archaeological application", en Lock, G., Stancic, G. (Eds.), Archaeology and Geographic Information Systems: A European Perspective, Londres, CRC Press, 171-186.

VV.AA. (2016): IMA 2015. Informe de Medio Ambiente en Andalucía, Consejería de Medio Ambiente y Ordenación del Territorio de la Junta de Andalucía.

VV.AA. (2011)Z Ecobarómetro de Andalucía (2011). Consejería de Medio Ambiente. Junta de Andalucía.

http://www.juntadeandalucia.es/medioambiente/portal web/web/temas ambientales/educacion y v v oluntariado ambiental/Sensibilizacion/Ecobarometro/EBA 2011 Informe completo.pdf.

(Consultado 20/08/2017). 
